\title{
Electrophysiology of executive control in spoken noun-phrase production: Dynamics of updating, inhibiting, and shifting
}

\author{
Katarzyna Sikora ${ }^{\mathrm{a}, *}$, Ardi Roelofs ${ }^{\mathrm{a}}$, Daan Hermans ${ }^{\mathrm{b}, \mathrm{c}}$ \\ ${ }^{a}$ Radboud University, Donders Institute for Brain, Cognition and Behaviour, Centre for Cognition, Montessorilaan 3, 6525 HR, Nijmegen, The Netherlands \\ ${ }^{\mathrm{b}}$ Radboud University, Behavioural Science Institute, Montessorilaan 3, 6525 HR, Nijmegen, The Netherlands \\ ${ }^{\text {c } R o y a l ~ D u t c h ~ K e n t a l i s, ~ P e t r u s ~ D o n d e r s p l e i n ~ 1, ~} 5271$ AA, Sint-Michielsgestel, The Netherlands
}

\section{A R T I C L E I N F O}

\section{Article history:}

Received 15 September 2015

Received in revised form

22 December 2015

Accepted 29 January 2016

Available online 2 February 2016

Keywords:

language production

executive control

\begin{abstract}
A B S T R A C T
Previous studies have provided evidence that updating, inhibiting, and shifting abilities underlying executive control determine response time (RT) in language production. However, little is known about their electrophysiological basis and dynamics. In the present electroencephalography study, we assessed noun-phrase production using picture description and a picture-word interference paradigm. We measured picture description RTs to assess length, distractor, and switch effects, which have been related to the updating, inhibiting, and shifting abilities. In addition, we measured event-related brain potentials (ERPs). Previous research has suggested that inhibiting and shifting are associated with anterior and posterior N200 subcomponents, respectively, and updating with the P300. We obtained length, distractor, and switch effects in the RTs, and an interaction between length and switch. There was a widely distributed switch effect in the N200, an interaction of length and midline site in the N200, and a length effect in the P300, whereas distractor did not yield any ERP modulation. Moreover, length and switch interacted in the posterior N200. We argue that these results provide electrophysiological evidence that inhibiting and shifting of task set occur before updating in phrase planning.
\end{abstract}

(c) 2016 Elsevier Ltd. All rights reserved.

\section{Introduction}

Fluent language production is important for successful communication. An average speaker can produce some 150 words per minute with as little as about one error every 1000 words (Levelt, 1989). Although speaking is a highly practiced psychomotor skill, it requires executive control (e.g., de Zubicaray, Wilson, McMahon, \& Muthiah, 2001; Roelofs, 2003; Roelofs \& Piai, 2011; Schnur, Schwartz, Kimberg, Hirshorn, Coslett, \& Thompson-Schill, 2009). Executive control refers to the regulative processes that ensure that our thoughts and actions are in accordance with our goals (e.g., Baddeley, 1996; Gilbert \& Burgess, 2008; Logan, 1985; Posner, 2012). According to an influential proposal (Miyake, Friedman, Emerson, Witzki, Howerter, \& Wager, 2000), executive control includes updating and monitoring of working memory representations (updating), inhibiting of unwanted responses (inhibiting), and shifting between tasks or mental sets (shifting). The updating ability determines working memory capacity (cf. Schmiedek, Hildebrandt, Lövdén, Wilhelm, \& Lindenberger, 2009).

\footnotetext{
* Corresponding author: Katarzyna Sikora, Radboud University, Donders Institute for Brain, Cognition and Behaviour, Centre for Cognition, Spinoza Building B.02.40, Montessorilaan 3, 6525 HR Nijmegen, The Netherlands.

E-mail address: K.Sikora@donders.ru.nl (K. Sikora).
}

Previous behavioral studies have shown that the updating, inhibiting, and shifting abilities determine the response time (RT) of picture naming and picture description (e.g., Piai \& Roelofs, 2013; Shao, Roelofs, \& Meyer, 2012; Sikora, Roelofs, Hermans, \& Knoors, 2015). However, little is known about the electrophysiological basis and dynamics of these abilities in language production. The aim of the present study was to obtain electrophysiological evidence on the dynamics of the involvement of updating, inhibiting, and shifting in spoken noun-phrase production.

Below, we first briefly review the RT evidence on the contributions of updating, inhibiting, and shifting to language production (Section 1.1). Next, we describe previous evidence on event-related brain potentials (ERPs) that inhibiting and shifting are generally associated with modulations of anterior and posterior N200 subcomponents, respectively, and updating with modulations of the P300 (Section 1.2). Then, we describe our experimental procedure, which consisted of overt noun-phrase production to describe pictures and a picture-word interference paradigm (Section 1.3). The procedure allows for measuring length, distractor, and switch effects, which have been shown to reflect the updating, inhibiting, and shifting abilities, respectively. In the remainder of this article, we report a study examining these effects in RTs and ERPs in language production (Sections 2 and 3). Finally, we discuss what our electrophysiological findings reveal 
about the dynamics of executive control in noun-phrase production (Section 4).

\subsection{Contributions of updating, inhibiting, and shifting to language production}

Theoretically, the updating, inhibiting, and shifting abilities are expected to contribute to language production. The updating ability is needed because speakers must keep in mind the intended goal of the conversation, monitor their performance and update the content of working memory while engaging in conceptual and linguistic processes (e.g., Levelt, 1989; Levelt, Roelofs, \& Meyer, 1999; Piai \& Roelofs, 2013). Moreover, the inhibiting ability is required to suppress incorrect names that are co-activated during lexical selection (e.g., Shao, Roelofs, Acheson, \& Meyer, 2014). Furthermore, the shifting ability is needed to switch between planning one type of phrase to another or switch from planning an utterance to monitoring the articulatory output (e.g., Levelt, 1989).

Recent studies have provided RT evidence that the updating, inhibiting, and shifting abilities contribute to language production. Shao et al. (2012) observed that updating ability was correlated with the mean RT of action naming but not of object naming, while inhibiting ability was correlated with the mean RT of both object and action naming. Action naming is typically more demanding than object naming. Thus, the correlation between updating ability and the mean RT of action but not object naming suggests that the engagement of updating is particular evident in demanding situations. Whereas Shao et al. (2012) obtained no correlation between updating ability and the mean RT of object naming, Piai and Roelofs (2013) observed that in a more demanding situation, namely picture naming during dual-task performance, the updating ability correlated with the mean RT of object naming. Shao et al. did not find a contribution of shifting to picture naming, but this may be due to the absence of some kind of switching in simple naming. To examine the contributions of updating, inhibiting, and shifting in a situation that requires actual switching, Sikora et al. (2015) asked participants to describe pictures of simple objects by producing noun phrases in Dutch. We describe the design and results of this study in some detail, because a similar design was used in the ERP study presented below.

In the study of Sikora et al. (2015), pictures were presented in color or black-and-white. In response to the colored pictures, participants produced determiner-adjective-noun phrases with the adjective referring to the color (e.g., "de groene vork", the green fork), the long phrases. In response to the black-and-white pictures, they produced determiner-noun phrases without the adjective (e.g., "de vork", the fork), the short phrases. In both cases, the determiner was a definite article, de or het in Dutch (and in another condition, not included in the present study, it was an indefinite article). In this task, the updating ability is needed because in planning the noun phrases, speakers have to conceptually identify the pictured object and retrieve from long-term memory a corresponding noun. For the long phrases, also the color needs to be identified and an adjective has to be retrieved from memory. Moreover, the appropriate gender-marked article needs to be retrieved. Following this, a syntactic structure has to be chosen and the determiner and noun, as well as the adjective for the long phrases, have to be serially ordered. Conceptual preparation and syntactic encoding are followed by morphophonological and phonetic encoding, and finally, articulation. The conceptual and linguistic processes require working memory (e.g., Levelt, 1989).

Given that more information needs to be derived from the picture, accessed in long-term memory, and manipulated in working memory for the long phrases than for the short phrases, the updating ability should be more strongly engaged when producing the long phrases. Consequently, the magnitude of the difference in RT between these phrase types, the length effect, was expected to reflect a speaker's updating ability. To assess the contribution of the inhibiting ability, the pictures were combined with auditory distractor words, which could be congruent (i.e., the name of the picture, e.g., vork) or incongruent (the name of another, semantically related picture, e.g., bord, plate). The inhibiting ability was expected to be more strongly engaged with incongruent than congruent distractors. Consequently, the magnitude of the difference in RT between these distractor types, the distractor effect, was expected to reflect a speaker's inhibiting ability. To assess the contribution of the shifting ability, the required phrase type (long or short) changed every second trial. Thus, two short phrases (for pictures in black-and-white) were followed by two long phrases (for pictures in color) and vice versa. A trial that repeats the previous phrase type (short preceded by short or long preceded by long) is a repeat trial, and a trial that does not repeat the previous phrase type (short preceded by long or long preceded by short) is a switch trial. Speakers need to engage the shifting ability on switch trials to enable to production of a different phrase type. The shifting ability should be more strongly engaged on switch than repeat trials. Consequently, the magnitude of the difference in RT between these trial types, the switch effect, was expected to reflect a speaker's shifting ability. In addition to picture description RTs, the participants' updating, inhibiting, and shifting abilities were measured using standard tasks to assess executive control. The operation-span and odd-one-out tasks (Conway et al., 2005) were used to assess verbal and nonverbal updating ability, respectively, the stop-signal task (Verbruggen, Logan, \& Stevens, 2008) to assess nonverbal inhibiting ability, and the shape-color switching task (Miyake et al., 2000) to assess nonverbal shifting ability.

It was found that participants described the pictures slower in the long phrase than in the short phrase condition (the length effect), slower in the incongruent than in the congruent distractor condition (the distractor effect), and slower in the switch than in the repeat condition (the switch effect). The length effect in the RTs correlated with the verbal but not the nonverbal updating scores, while the distractor effect correlated with the inhibiting scores. No correlation was found between the switch effect in the mean RTs and the shifting scores. However, the shifting scores correlated with the switch effect in the normal part of the underlying RT distribution. These results suggest that updating, inhibiting, and shifting each influence the speed of phrase production, thereby demonstrating a contribution of all three executive control subabilities to language production.

A switch effect was obtained for the short phrases but not for the long phrases. This observation corresponds to the asymmetry in switch costs that is often obtained in task switching (e.g., Allport \& Wylie, 1999, 2000; Gilbert \& Shallice, 2002; Yeung \& Monsell, 2003) and language switching (e.g., Jackson, Swainson, Cunnington \& Jackson, 2001; Meuter \& Allport, 1999). According to Allport and Wylie (1999, 2000), the asymmetrical switch effect is caused by differential task-set inertia, which refers to the idea that the irrelevant task set of the previous trial is still active on the current trial and needs to be actively disengaged. Colored pictures allow as responses both long phrases and short phrases, whereas black-and-white pictures only allow short-phrase responses. Therefore, to prevent inadvertent short-phrase responses to colored pictures, the task set for the short phrases may be inhibited and the task set for the long phrases may be enhanced. In contrast, on trials with black-and-white pictures, inhibition of long phrases and enhancement of short phrases is not needed. As a consequence, disengagement from the previous task set will take much longer in switching to short phrases than to long phrases, as observed by Sikora et al. (2015). One of the aims of the present ERP 
study was to replicate the behavioral observation of an asymmetric switch cost and to assess its electrophysiological manifestation.

\subsection{ERP manifestations of updating, inhibiting, and shifting}

Although previous behavioral studies have shown that updating, inhibiting, and shifting determine the RT of picture naming and description, there is a lack of evidence on the electrophysiological basis and dynamics of these abilities in language production. Previous research has identified electrophysiological correlates of executive control (e.g., Brydges, Fox, Reid, \& Anderson, 2014; Folstein \& Van Petten, 2008; Jackson et al., 2001; Kok, 2001; Polich \& Kok, 1995; Polich, 2007). More specifically, the N200 and P300 components of the ERP have been studied extensively in relation to executive functions. However, these studies typically used rather simple manual tasks like oddball, Eriksen flanker, and Simon, or simple picture naming. Moreover, the studies typically concentrated on one executive function at a time, like updating, inhibiting, or shifting, but did not examine all three functions simultaneously using a single design. Furthermore, it has remained unclear how the N200 and P300 components of the ERP reflect the updating, inhibiting, and shifting abilities in a more complex task like phrase production.

The N200 component of the ERP is a negative-going deflection observed approximately 200-350 ms after stimulus onset, although the time window can vary depending on several factors, including characteristics of the stimuli, task, and participants (e.g., Folstein \& Van Petten, 2008). The N200 can be subdivided into anterior and posterior subcomponents (e.g., Folstein \& Van Petten, 2008; Verhoef, Roelofs, \& Chwilla, 2010). The anterior N200 typically has a maximal amplitude at frontocentral electrode sites (i.e., $\mathrm{Fz}$ and $\mathrm{Cz}$ ). Anterior N200 modulations have been found in various tasks involving inhibitory control such as the go/no-go task (e.g., Jodo \& Kayama, 1992; Schmitt, Münte, \& Kutas, 2000), the flanker task (e.g., Heil, Osman, Wiegelmann, Rolke, \& Hennighausen, 2000; Kopp, Rist, \& Mattler, 1996), and the stop-signal task (e.g., Schmajuk, Liotti, Busse, \& Woldorff, 2006). A larger N200 amplitude has been associated with greater inhibitory demand. The anterior N200 has been linked also to inhibition in language switching (e.g., Jackson et al., 2001; Verhoef, Roelofs \& Chwilla, 2009).

Moreover, Verhoef et al. (2010) obtained evidence that switching between languages is also reflected in the posterior N200, which typically has a maximal amplitude at centroparietal electrode sites (i.e., $\mathrm{Cz}$ and $\mathrm{Pz}$ ). In testing bilingually unbalanced participants, Verhoef et al. found switch costs in the posterior N200 for the second language (L2) but not for the first language (L1). This difference in modulation of the posterior N200 was taken to reflect differential difficulty of disengagement from the previous language. Presumably, in unbalanced bilinguals, disengaging the weaker L2 is easier than disengaging the stronger L1. Consequently, the posterior N200 may reflect an effect for L2 switch trials requiring disengagement of the stronger L1, but not for L1 switch trials requiring disengagement of the weaker L2. Note that this account assumes that there was no differential inhibition of languages, otherwise the switch effect in the posterior N200 should have been obtained for L1 rather than L2 (i.e., switching to L1 would have required overcoming previous inhibition). In line with this assumption, the amplitude of the anterior N200 did not differ between languages in the study of Verhoef et al. We are not aware of other studies testing for a switching effect in the posterior N200. The present study further examined this effect.

The P300 component of the ERP is a positive-going deflection observed approximately 200-500 ms after stimulus onset, although the time window can vary depending on several factors, including characteristics of the stimuli, task, and participants (e.g., Kok, 2001; Polich \& Kok, 1995; Polich, 2007). The P300 can be divided into two subcomponents: P3a and P3b. The P3a has a central peak at the scalp and seems to reflect involuntary shifts or allocation of perceptual attention. For instance, the P3a is elicited by infrequent distractor stimuli in an oddball task. In the oddball task, participants are presented with a series of frequent standard stimuli and infrequent target and distractor stimuli, whereby a response is required only for the targets. In contrast to the P3a, the P3b has a more central-parietal distribution and is associated with the voluntary allocation of attention and updating of working memory. The P3b occurs in response to infrequent targets in the oddball task but also in tasks requiring more complex types of information processing, such as N-back (e.g., Evans, Selinger, \& Pollak, 2011; Watter, Geffen, \& Geffen, 2001) and dual-task performance (e.g., Kramer, Sirevaag, \& Braune, 1987; Strayer \& Drews, 2007; Wickens, Kramer, Vanasse, \& Donchin, 1983). Previous research showed that the amplitude of the P3b component is modulated by the relative demands on the updating of working memory. More difficult task conditions elicit a smaller P3b amplitude than less demanding conditions (Evans et al., 2011; Kramer et al., 1987; Kok, 2001; Polich, 2007; Strayer \& Drews, 2007; Watter et al., 2001; Wickens et al., 1983). A decreased P3b amplitude reflects a greater updating demand. A relation between the amplitude of the P3b component and updating ability has been found in both adults and children (Brydges et al., 2014; Evans et al., 2011). Given that we expect to find a modification of the P3b but not the P3a, we refer to the P3b as P300 in the remainder of this article. Previous studies that investigated phrase and sentence production have also found modulations of the P300 that were related to the demands on the updating of working memory (Marek, Habets, Jansma, Nager, \& Münte, 2007; Habets, Jansma, \& Münte, 2008). However, in these studies, the P300 amplitude was larger in more difficult than in easier conditions, different from the results using non-linguistic tasks referred to above. We further discuss this issue in Section 4.2 below.

It is unclear whether shifting is reflected in the P300. Using tasks with binary manual responses, some prior studies have obtained P300 switching effects (Karayanidis, Coltheart, Michie, \& Murphy, 2003; Lorist, Klein, Nieuwenhuis, De Jong, Mulder, \& Meijman, 2000; Rushworth, Passingham, \& Nobre, 2002), whereas other studies did not find an effect (Hsieh, 2006; Hsieh \& Yu, 2003; Hsieh \& Liu, 2005; Poulsen, Luu, Davey, \& Tucker, 2005). The present study examined whether the amplitude of the P300 is modulated by shifting in spoken phrase production.

\subsection{Outline of the present study}

Following Sikora et al. (2015), we assessed language production performance using a picture description task and a picture-word interference procedure. We measured ERPs to assess how length, distractor, and switch effects are reflected in the N200 and P300 components. More specifically, based on the literature (reviewed in Section 1.2), we expected that the distractor and switch effects would be reflected in the anterior and posterior N200 subcomponents, respectively, and the length effect in the P300. Moreover, we expected a length effect in the anterior N200 (i.e., more inhibition during the production of long than short phrases) and an interaction between length and switch in the posterior N200 (harder shifting to short than long phrases due to the differential inhibition). Additionally, we expected that the magnitude of the ERP effects would correlate with the magnitude of the corresponding RT effects. Theoretically, a speaker must shift to the relevant task set (e.g., to produce a long phrase) and inhibit an irrelevant task set (to produce a short phrase) before the required 
phrase can actually be planned, which requires the updating ability. This would imply that the shifting and inhibiting abilities are engaged before the updating ability, in line with the association of shifting and inhibiting with the N200 and updating with the P300.

\section{Method}

\subsection{Participants}

Twenty-eight native speakers of Dutch participated in the experiment $(23$ women and 5 men, mean age $=23.75$ years, age range: 19 to 34 years). The participants were recruited via the Radboud University SONA system. They received 25 Euros or 2.5 credit for their participation.

\subsection{Design and behavioral procedure}

The participants signed the informed consent before the experimental session. After the experimental session, participants were debriefed and paid for their participation. An experimental session lasted about 2 hour and 30 minutes.

Participants had to describe a picture presented in the middle of a computer screen while trying to ignore a spoken distractor that was played via headphones. Each trial began with a fixation cross which remained on the screen for $700 \mathrm{~ms}$, followed by the presentation of a spoken distractor and a picture simultaneously (i.e., with the same presentation onset). The picture remained on the screen for $250 \mathrm{~ms}$ followed by a blank screen for $2450 \mathrm{~ms}$. To concentrate on the influences of executive control and to reduce variance due to differences in processing speed among pictures and words, only a limited number of objects and colors was used. The set of stimuli consisted of four pictures, namely a bottle, a plate, a glass, and a fork, and four spoken distractors, which were the names of these objects. All spoken distractors were monosyllabic Dutch words, which were all semantically related (like in the color-word Stroop task, e.g., Roelofs, 2003). Two nouns had non-neuter grammatical gender, taking the definite article de, and two nouns had neuter gender, taking the definite article het. Different from Sikora et al. (2015), the experiment did not have an indefinite-phrase condition. There were two practice blocks and ten experimental blocks of trials. Participants had to describe all the pictures using noun phrases with a definite article and ignore the spoken distractors. Each experimental block consisted of 32 trials. In total there were 320 trials. We used the program Mix (Van Casteren \& Davis, 2006) to randomize our stimulus lists. A restriction on the randomization was that stimuli were not allowed to repeat on consecutive trials.

The picture names and the spoken distractors could be either two identical nouns (congruent condition) or two different nouns taking the same grammatical gender (incongruent condition). These distractor conditions had the same number of trials. The pictures where either black-and-white drawings or colored drawings, either blue or green. The participants were instructed to produce determiner-noun phrases (e.g., "de vork", the fork) when the presented picture was a black-and-white drawing, the short phrase condition. When the picture was presented in one of the two colors, the participants had to produce a phrase that included an article, a color adjective, and the name of the object (e.g., "de groene vork", the green fork), the long phrase condition. The number of trials for these conditions was the same. The pictures were presented such that the required phrase type changed every second trial. Thus, in the trial sequence, two black-and-white pictures were followed by two colored pictures, which were followed by two black-and-white pictures, etc. The number of trials for the repeat and switch conditions was equal.

\subsection{EEG procedure}

The EEG was recorded from 32 cap-mounted active $\mathrm{Ag} / \mathrm{AgCl}$ electrodes. Additionally, four electrodes were used to monitor horizontal and vertical eye movements and two electrodes were used to monitor mouth movements. Moreover, two electrodes were placed on the right and left mastoid bones. The signal was amplified with BrainAmps DC amplifiers with a $125 \mathrm{~Hz}$ low-pass filter, $500 \mathrm{~Hz}$ sampling frequency and a time constant of $10 \mathrm{~s}$. Electrode impedance of the recording was kept below $5 \mathrm{k} \Omega$. All electrodes were referenced on-line to the $\mathrm{FCz}$ electrode and rereferenced off-line to averaged mastoids.

\subsection{RT analysis}

The data of six participants were excluded from the analysis due to technical errors in the EEG recording. In total, the data of 22 subjects were included in the analysis. Moreover, responses were excluded from the analysis if the produced phrase did not match the correct phrase or when the response included any kind of disfluency or was not completed before the end of a trial. Mean RTs were calculated for long phrase, short phrase, congruent distractor, incongruent distractor, repeat trials, and switch trials. Repeated measures ANOVAs were conducted to test for main effects and interactions between conditions. Three effects were defined: length (short vs. long phrase), distractor (congruent vs. incongruent), and switch (repeat vs. switch trials).

\subsection{EEG analysis}

EEG analyses were performed using the software Brain Vision Analyzer (Brain Products, Germany). Trials excluded from the RT analysis were also discarded from the EEG analysis. The EEG electrodes were re-referenced to the averaged right and left mastoids. Single waveforms were filtered with a $30 \mathrm{~Hz}, 12 \mathrm{~dB}$ lowpass filter. Thereafter, segments starting $200 \mathrm{~ms}$ before and 600 ms after stimulus onset were formed. The segments were baseline-corrected using the 200-ms window before stimulus onset, and they were screened for eye movements, electrode drifting, and EMG artifacts with a $75-\mu$ Volt criterion. After the artifact rejection, there were at least 30 trials left per participant per condition. Next, averaged ERPs were computed per participant and per condition.

The effects of length, distractor, and switch were evaluated at the midline electrodes (Fz, Cz, Pz) for N200 and P300 modulations. Based on the literature and visual inspection of the waveforms, the time windows for the $\mathrm{N} 200$ and $\mathrm{P} 300$ were, respectively, from 250 $\mathrm{ms}$ to $400 \mathrm{~ms}$ (N200) and from $400 \mathrm{~ms}$ to $600 \mathrm{~ms}$ (P300). A repeated measures ANOVA was conducted for each of the time windows. Moreover, four quadrants were defined: right anterior (F4, F8, FC2, FC6, C4), left anterior (F3, F7, FC1, FC5, C3), right posterior (CP2, CP6, P4, P8, O2), and left posterior (CP1, CP3, P3, P7, 01). The effects of length, distractor, and switch were also evaluated for the four quadrants. In addition, we analyzed correlations among the three main effects in the RTs and in the ERPs at the midline sites ( $\mathrm{Fz}, \mathrm{Cz}, \mathrm{Pz}$ ).

\section{Results}

\subsection{Behavioral performance}

Mean reaction times and error rates for each of the conditions are presented in Table 1 . Mean RTs were longer for the incongruent than the congruent condition (distractor effect), longer for 
Table 1

Mean Reaction Times (Milliseconds) and Percentage Error (Between Parentheses) in the Picture-Description Task.

\begin{tabular}{|c|c|c|c|c|}
\hline \multirow[t]{2}{*}{ Distractor } & \multirow[t]{2}{*}{ Length } & \multicolumn{2}{|l|}{ Switch } & \multirow[b]{2}{*}{ Total } \\
\hline & & Repeat & Switch & \\
\hline \multirow[t]{3}{*}{ Congruent } & Long & $712(12)$ & $697(14)$ & 705 (13) \\
\hline & Short & $644(6)$ & $679(6)$ & $660(6)$ \\
\hline & Total & $678(9)$ & $687(10)$ & $683(9)$ \\
\hline \multirow[t]{3}{*}{ Incongruent } & Long & 748 (17) & 742 (19) & 745 (18) \\
\hline & Short & $689(9)$ & $710(9)$ & $698(9)$ \\
\hline & Total & 717 (13) & $726(14)$ & $722(14)$ \\
\hline \multirow[t]{3}{*}{ Total } & Long & $730(15)$ & $720(16)$ & 725 (15) \\
\hline & Short & $665(7)$ & $693(7)$ & $679(7)$ \\
\hline & Total & $698(11)$ & 707 (12) & $702(11)$ \\
\hline
\end{tabular}

the long phrase than for the short phrase condition (length effect), and longer for the switch condition than for the repeat condition (switch effect).

The statistical analysis of the RTs revealed that there was a main effect of distractor, indicating that participants were slower in the incongruent than in the congruent condition, $F(1,21)=$ 50.98, $M S E=33684, p<.001, \eta_{\mathrm{p}}{ }^{2}=.71$. There was also a main length effect, indicating that participants were slower in the long phrase than in the short phrase condition, $F(1,21)=12.06$, $M S E=46877, p<.01, \eta_{\mathrm{p}}{ }^{2}=.37$. We also found a main switch effect, indicating that participants were slower in the switch than in the repeat condition, $F(1,21)=6.42, M S E=1657, p<.05, \eta_{\mathrm{p}}{ }^{2}=.23$.

Additionally, we found an interaction between length and switch, $F(1,21)=16.53, M S E=16717, p<.001, \eta_{\mathrm{p}}^{2}=.44$. Fig. 1 shows the RT pattern. Post-hoc analysis revealed that the switch effect was present for the short phrases, $t=5.42, p<.001$, but not for the long phrases, $t=-1.66, p=.112$. There were no other interactions.

To summarize, the present study obtained length, distractor, and switch effects, and an interaction between length and switch. These behavioral findings replicate Sikora et al. (2015).

\subsection{Electrophysiological findings}

Fig. 2 presents grand-averaged ERP waveforms at the three midline electrodes (Fz, Cz, Pz) for the switch (repeat vs. switch), distractor (incongruent vs. congruent), and length (short vs. long

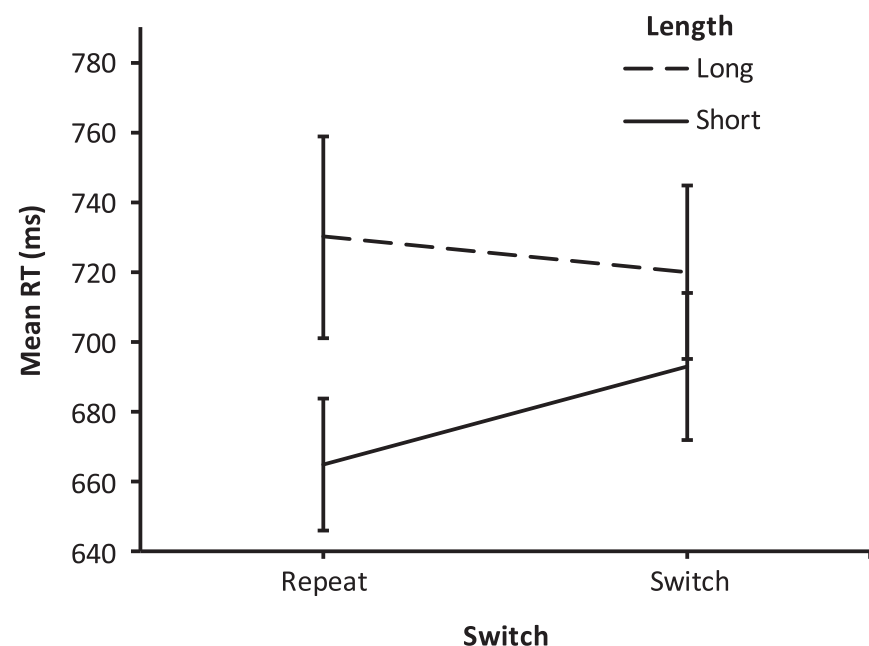

Fig. 1. Mean response time (RT) for the picture descriptions per length condition (short, long) on repeat and switch trials. The error bars indicate one standard error. phrase) conditions. Fig. 3 presents topographic maps for the switch, distractor, and length effects for the $0-250 \mathrm{~ms}, 250-400 \mathrm{~ms}$ (N200), and 400-600 ms (P300) time windows. For the 0-250 ms time window, no significant effects were obtained.

\subsubsection{0-400 ms (N200) window}

At the midline sites $(\mathrm{Fz}, \mathrm{Cz}, \mathrm{Pz})$, we found a main switch effect, $F$ $(1,21)=15.89, M S E=338.25, p<.001, \eta_{\mathrm{p}}{ }^{2}=.43$. There was a larger negative-going ERP amplitude for the switch trials than for the repeat trials. Moreover, we found no main effect of length, but there was an interaction between electrode and length, $F(1,21)=$ $14.45, M S E=95.60, p<.001, \eta_{\mathrm{p}}{ }^{2}=.41$. Post-hoc analysis revealed that there was a significant length effect at the frontal electrode (Fz), $t=-2.34, p<.05$, with the N200 amplitude being larger for the long phrases than for the short phrases (anterior N200). There was also a significant length effect at the parietal electrode $(\mathrm{Pz})$, $t=2.45, p<.05$, with the N200 amplitude being smaller for the long phrase condition than for the short phrase condition (posterior N200). At the central electrode (Cz), the length effect was not significant, $t=-1.0, p=.34$. Thus, the length effect in the N200 changed direction between frontal and parietal electrodes (i.e., from long $>$ short to long $<$ short).

The quadrant analysis also revealed a main switch effect, $F(1$, $21)=18.29, M S E=251.74, p<.001, \eta_{\mathrm{p}}^{2}=.47$. The ERP amplitude was more negative-going for the switch trials than for the repeat trials. Moreover, we found an interaction between quadrant and length, $F(3,63)=12.40, M S E=52.49, p<.001, \eta_{\mathrm{p}}{ }^{2}=.37$. Post-hoc analysis revealed that the length effect in the N200 was present for the posterior right quadrant, $t=3.00, p<.01$, and posterior left quadrant, $t=2.30, p<.05$, but not for the anterior quadrants. For both posterior quadrants, there was a more negative-going ERP amplitude for the short phrase condition than for the long phrase condition, in line with the midline analysis. We also found an interaction between quadrant, length, and switch, $F(3,63)=3.50$, $M S E=9.07, p<.05, \eta_{\mathrm{p}}{ }^{2}=.14$. Post-hoc analysis revealed that the interaction between length and switch was present for the posterior right quadrant, $F(1,21)=10.15, M S E=27.34, p<.01, \eta_{\mathrm{p}}{ }^{2}=.33$, and for the posterior left quadrant, $F(1,21)=4.86, M S E=20.76$, $p<.05, \eta_{\mathrm{p}}{ }^{2}=.19$, but not for the anterior right quadrant, $F(1$, $21)=.001, M S E=0.01, p=.97, \eta_{\mathrm{p}}{ }^{2}=.00$, and for the anterior left quadrant, $F(1,21)=.05, M S E=.42, p=.83, \eta_{\mathrm{p}}^{2}=.002$. For the posterior right quadrant, switch and repeat trials did not differ for the long phrase condition, $t=-.96, p=.35$, while in the short phrase condition there was a more negative $\mathrm{N} 200$ for the switch than for the repeat trials, $t=-4.88, p<.001$. Similar, for the posterior left quadrant, switch and repeat trials did not differ for the long phrase condition, $t=-1.29, p=.21$, while in the short phrase condition there was a more negative $\mathrm{N} 200$ for the switch than for the repeat trials, $t=-3.72, p<.001$.

\subsection{2. $400-600 \mathrm{~ms}$ (P300) window}

At the midline sites $(\mathrm{Fz}, \mathrm{Cz}, \mathrm{Pz})$, we found a significant main effect of length, $F(1,21)=8.0, M S E=267, p<.01, \eta_{\mathrm{p}}{ }^{2}=.27$. In particular, there was a smaller positive-going ERP amplitude for the long than for the short phrase condition.

We did not find any significant main effects for the quadrant analysis in the late time window. However, the main length effect was marginally significant, $F(1,21)=3.74, M S E=96.15, p=.07$, $\eta_{\mathrm{p}}{ }^{2}=.15$. The positive ERP amplitude was smaller for the long than for the short phrases.

To summarize, the ERP findings suggest the following time course of effects. In the N200 time window (250-400 ms), a switch effect is obtained that is widely distributed across the scalp. Switch trials yield a larger negativity than repeat trials. Moreover, the switch effect interacts with length at posterior electrode sites (posterior N200). The larger negativity for switch than repeat trials 


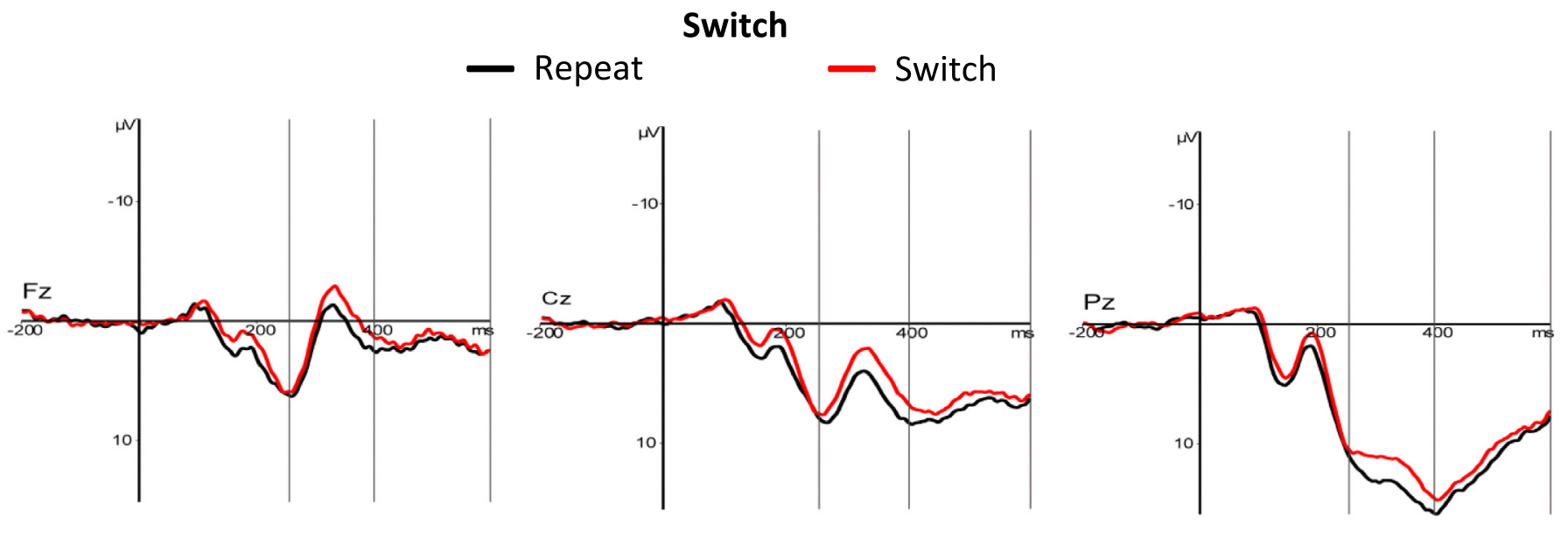

\section{Distractor}
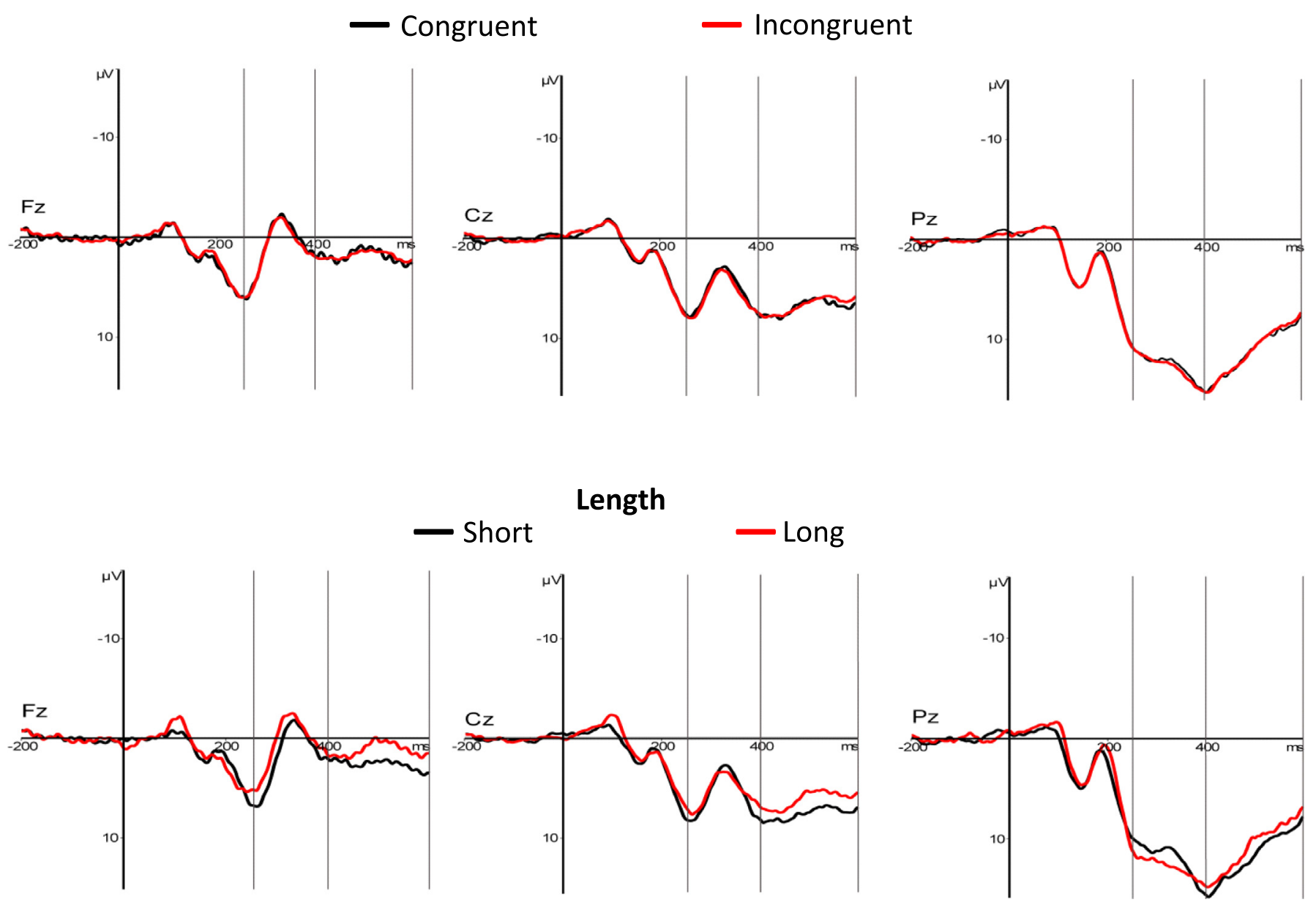

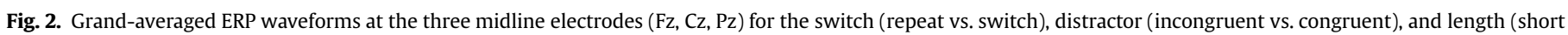
vs. long) conditions. The gray vertical lines indicate the $250-400 \mathrm{~ms}$ (N200) and 400-600 ms (P300) time windows.

is obtained for the short phrases but not for the long phrases. Length interacts with electrode at midline sites: The N200 is larger for the long than short phrases at the frontal site (anterior N200) and smaller for long than short at the parietal site (posterior $\mathrm{N} 200$ ). In the $\mathrm{P} 300$ time window (400-600 ms), only a main effect of length is obtained. A smaller positivity is obtained for the long phrases than for the short phrases. Distractor does not yield an effect in any of the time windows.

\subsection{Correlations between the electrophysiological and behavioral responses}

We analyzed the correlations among the three effects (length, distractor, switch) in the RTs and in the ERPs. We conducted the analysis at the midline electrodes for the time windows of 250$400 \mathrm{~ms}$ and $400-600 \mathrm{~ms}$. We tested for correlations between the distractor and switch effects in the RTs and the N200, and between 


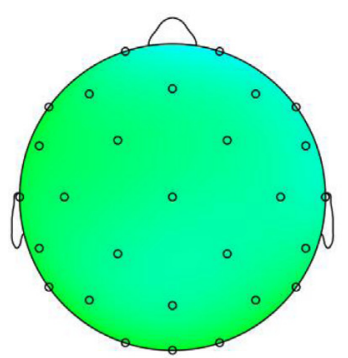

\section{Switch minus repeat}

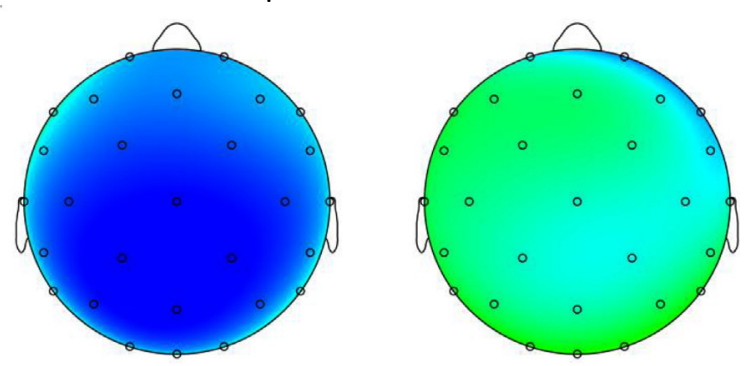

\section{Incongruent minus congruent}
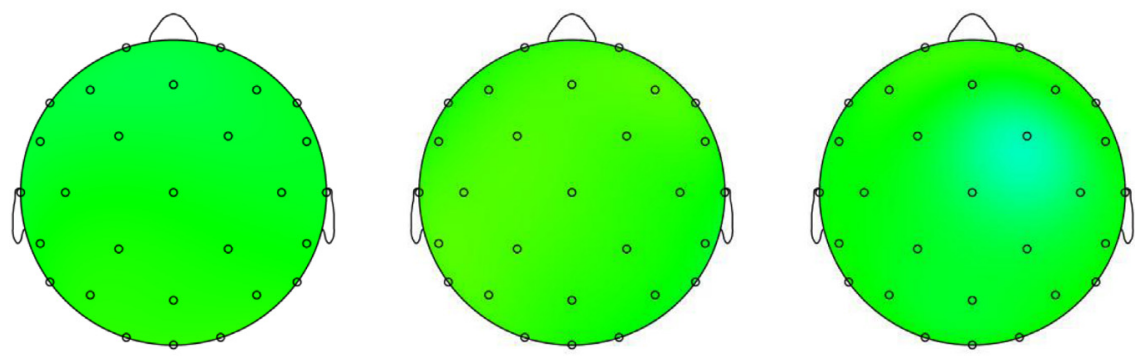

\section{Short minus long}
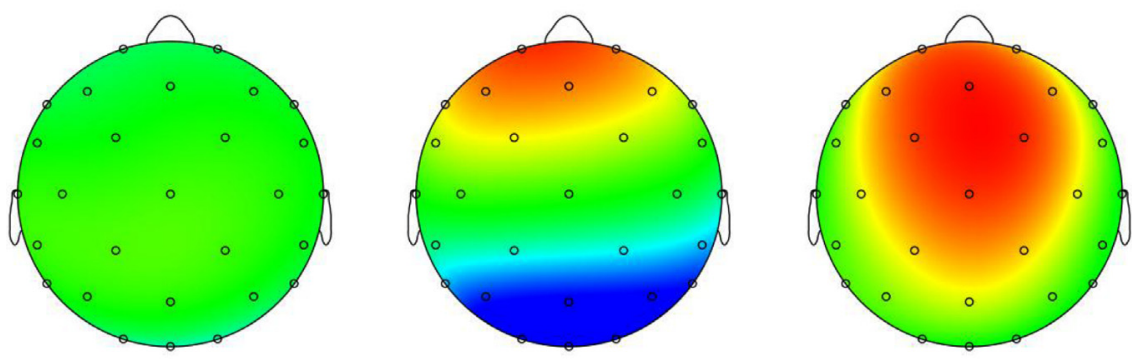

$0-250 \mathrm{~ms}$

$250-400 \mathrm{~ms}$

$400-600 \mathrm{~ms}$

\section{$-1.5 \mu \mathrm{V} \quad 0 \mu \mathrm{V} \quad 1.5 \mu \mathrm{V}$}

Fig. 3. Topographic maps for the switch, distractor, and length effects for the $0-250 \mathrm{~ms}, 250-400 \mathrm{~ms}$ (N200), and 400-600 ms (P300) time windows.

the length effect in the RTs and the P300. In the N200 time window, we found a significant negative correlation between the magnitude of the switch effect in the RTs and the ERPs at the frontal electrode (Fz), $r=-.39, p<.05$, but we found no correlation for distractor. In the P300 time window, we found a significant negative correlation between the magnitude of the length effect in the RTs and ERPs at the parietal $(\mathrm{Pz})$ and at the central $(\mathrm{Cz})$ electrodes, $r=-.61, p<.01$, and $r=-.41, p<.05$, respectively.

\section{Discussion}

The current study investigated the electrophysiological basis and dynamics of updating, inhibiting, and shifting in noun-phrase production. We measured how an increase in demand on updating, inhibiting, and shifting modulated noun-phrase production RTs and electrophysiological components, in particular, the N200 and P300. Our RT and ERP results generally confirmed the expectations outlined in Section 1.3, except that we found a distractor effect only in RTs but not in ERPs. We obtained switch effects in RTs and the N200, which was widely distributed across the scalp. The amplitude of the N200 was larger for switch than for repeat trials. Moreover, the switch effect interacted with length at posterior electrode sites and in RTs. As expected, the amplitude of the posterior N200 was larger for switch than repeat trials for the short phrases but not for the long phrases, and the RT switch effect was present for the short but not for the long phrases. This is in line with the assumption that in switching between phrase types, it is more difficult to overcome previous inhibition in switching to short phrases (previously inhibited) than to long phrases (not inhibited). We also obtained evidence for the differential inhibition of long and short phrases. In line with the assumption that the task set for short phrases is inhibited during the production of long phrases, the amplitude of the anterior N200 was larger for the long than the short phrases. In the posterior N200, the reverse effect was obtained, suggesting greater difficulty in overcoming previous inhibition for short than long phrases. Finally, we obtained a length effect in RTs and the P300. The P300 amplitude was smaller on long- than short-phrase trials, in line with the assumption of greater engagement of the updating ability on longthan short-phrase trials.

We argued that, theoretically, speakers must shift to the relevant task set (e.g., to produce a long phrase) and inhibit an 
irrelevant task set (to produce a short phrase) before the required phrase can actually be planned, which requires the updating ability. Based on this, we expected that the shifting and inhibiting abilities would be engaged before the updating ability, in line with the association of shifting and inhibiting with the N200 and updating with the P300. Our ERP results confirmed these expectations, providing evidence that inhibiting and shifting of task set are engaged before updating in phrase planning. In the remainder of this article, we further discuss these findings in more detail and relate them to existing findings in the literature. In particular, we further discuss the N200 effects (Section 4.1) and the P300 effect (Section 4.2).

\subsection{N200 effects}

We observed that widely distributed across the scalp, the N200 was larger for switch than repeat trials. Moreover, in the midline analysis, the N200 was larger for long than short phrases at the frontal electrode (anterior N200) and larger for short than long phrases at the parietal electrode (posterior N200). Moreover, in the posterior quadrants, the N200 was larger for switch than repeat trials for the short but not for the long phrases. The latter finding is in line with the interaction between length and switch obtained in the RTs.

Previous research has suggested that the anterior N200 is associated with inhibition and the posterior N200 with shifting. Our finding of a broadly distributed switch effect would suggest that switching between phrase types not only engages the shifting ability (which would be reflected at posterior sites) but also the inhibiting ability (which would be reflected at anterior sites). The involvement of inhibition in switching between phrase types agrees with evidence that inhibition is also involved in switching between languages (Jackson et al., 2001; Meuter \& Allport, 1999) and in task switching (Allport \& Wylie, 1999, 2000; for a review, see Koch, Gade, Schuch, \& Philipp, 2010). The asymmetrical switch cost in the RTs that we obtained (i.e., a switch effect for the short but not for the long phrases) corresponds to the asymmetrical switch costs often obtained in language switching and in task switching. For example, switch costs tend to be larger for the stronger language (L1) than the weaker language (L2) and larger for the stronger task (e.g., Stroop reading) than the weaker task (e.g., Stroop color naming). The asymmetrical switch cost is often interpreted in terms of differential task-set inertia. In performing the weaker language or task, the weaker task set should be enhanced or the competing task set for the stronger language or task should be inhibited. As a consequence, in switching to the stronger language or task, the previous inhibition should be overcome or the previous enhancement of the weaker task should be overcome. This prolongs RTs on switch trials for the stronger language or task.

Similarly, in producing long phrases, the task set for producing short phrases (allowed by a colored picture) needs to be inhibited, whereas in producing short phrases, the task set for producing long phrases (not allowed by a black-and-white picture) does not need to be inhibited. This explains the length effect we obtained for the anterior N200 (i.e., a lager N200 for long than short phrases). Presumably, more inhibition is needed on switch than repeat trials, but we obtained no interaction between length and switch in the anterior N200. However, this absence of an interaction may be due to insufficient power given that the effect was assessed for only one electrode (Fz). Similarly, the posterior interaction between length and switch (i.e., a switch effect for short but not for long phrases) was obtained in the quadrant analysis (which included several electrodes) but not in the midline analysis (which concerned only one posterior electrode, Pz). In the posterior quadrants, the N200 was larger for the short than the long phrases, in line with the assumption that in switching to a short phrase, the previous inhibition of its task set needs to be overcome.

We obtained a negative correlation between the switch effect in RTs and the anterior N200. This suggests that stronger engagement of the inhibiting ability reduces the RT switch effect. This is in line with the findings of Shao et al. (2014), who observed a similar negative correlation between name-agreement affects in the anterior N200 and picture naming RTs.

Contrary to our expectations, we did not find a distractor effect in the N200 component, and also no correlations. It is unclear why we failed to find the ERP distractor effect. We can exclude the possibility that the distractor manipulation was unsuccessful because we did find the distractor effect in RTs. Subjects responded slower on incongruent than congruent trials. A possible reason for the lack of an N200 distractor effect might be the modality of the distractors used in our study. Previous studies examining the electrophysiology of Stroop or Stroop-like tasks used visual distractors (e.g., Holmes \& Pizzagalli, 2008; Silton et al., 2010). In the current study, we used auditory distractors. In a previous ERP study examining semantic effects of spoken distractors in picture naming, Aristei, Melinger, and Abdel Rahman (2011) also obtained no significant distractor effect. Moreover, it has been observed that the N200 effect for no-go versus go trials in the stop-signal task is much smaller or absent if an auditory version of the task is used (e.g., Falkenstein, Hoormann, \& Hohnsbein, 1999). Thus, it seems possible that the use of auditory distractors in our study could be the reason why we did not find an N200 effect. However, further studies need to be carried out in order to investigate whether auditory distractors in Stroop-like tasks yield attenuated or no N200 modulations.

Taken together, our findings suggest that the greater executivecontrol demand on switch than repeat trials slows down phrase production and increases the N200 broadly across the scalp. Moreover, the larger anterior N200 for the long than for the short phrases suggests stronger involvement of inhibition on the longthan the short-phrase trials. As a consequence, a switch asymmetry is obtained in the posterior N200: For the short but not the long phrases previous inhibition need to be overcome. These findings point to a complex relation between inhibiting and shifting abilities in switching between phrase types in language production.

\subsection{P300 effect}

We found that the long phrases yielded longer RTs and a smaller P300 amplitude than the short phrases. In the short phrase condition, subjects had to process and maintain in working memory two parts of the phrase, the determiner and the noun, while in the long phrase condition they had to process and maintain three parts, namely the determiner, the adjective, and the noun. Thus, the long phrases required more updating of working memory than the short phrases, which was reflected in the longer RTs and the smaller amplitude of the P300. This finding is in line with previous research using non-linguistic tasks, which observed a smaller P300 amplitude in conditions with higher demand on updating (Evans et al., 2011; Watter et al., 2001). Moreover, the negative correlation between the length effects in the RTs and P300 suggests that greater engagement of the updating ability (i.e., a larger P300 effect) leads to a reduction of the length effect in the RTs.

However, previous studies that investigated phrase and sentence production have found increases of the P300 that were related to greater demand on the updating of working memory (Marek et al., 2007; Habets et al., 2008). In the study of Marek et al., participants produced phrases in easy, medium, and difficult 
conditions. In the easy condition, a phrase had to be produced to describe the direction of an arrow (e.g., "downwards"), in the medium condition, the phrase had to describe the direction and destination shape (e.g., "downwards to the triangle"), and in the difficult condition, the phrase had to describe the direction, destination shape, and its color (e.g., "downwards to the grey triangle"). Marek et al. found that the amplitude of the P300 was larger in the medium and difficult conditions than in the easy condition. In the study of Habets et al. (2008), participants had to produce sentences using temporal connectives (i.e., before or after) and verbs. The sentences had to be produced in response to sequentially presented objects (e.g., first a book was presented, and then a couch) in chronological order ("After I read (book), I sit (couch)") or non-chronological order (e.g., "Before I sit (couch), I read (book)"). Habets et al. observed that the amplitude of the P300 was larger in non-chronological than in the chronological condition, which was attributed to a greater demand on the updating of working memory in the more difficult, non-chronological condition. Thus, similar to our results, these studies show that differences in updating demand modulate the P300. However, whereas we have found a larger P300 in the easier condition (i.e., in producing the short phases), the studies by Marek et al. and Habets et al. found a smaller P300 in the easier condition. As indicated, our results are in agreement with previous non-linguistic studies showing that more demanding conditions elicit a smaller P300 amplitude than less demanding conditions (Evans et al., 2011; Kramer et al., 1987; Kok, 2001; Polich, 2007; Strayer \& Drews, 2007; Watter et al., 2001; Wickens et al., 1983).

It is unclear why a larger P300 amplitude reflects greater updating demand in some studies but a smaller demand in others. Kok (2001) suggested that the amplitude of the P300 may reflect the amount of capacity allocated to meet the task demands (i.e., a larger P300 means that more capacity is allocated) or the amount of capacity that is consumed (i.e., a larger P300 means that less capacity is consumed). If participants allocate more capacity in difficult than easier conditions to meet the task demands, and this difference is preserved despite differential consumption, then the P300 amplitude will be larger in difficult than easier conditions, in line with the results of Marek et al. (2007) and Habets et al. (2008). In contrast, if participants do not allocate more capacity in difficult than easier conditions, or if they do but this difference is counteracted by differential consumption, then the P300 amplitude will be smaller in difficult than easier conditions, in line with our results and those of several non-linguistic studies in the literature. Future research may further examine these possibilities. Important for now is that we observed that differences in updating demand modulate the P300, in line with what Marek et al. and Habets et al. found for phrase and sentence production, and what other studies found for non-linguistic tasks.

We found no switch effect in the amplitude of the P300. The evidence in the literature for task switching effects on the P300 is mixed. Whereas some studies have reported P300 switching effects (Karayanidis et al., 2003; Lorist et al., 2000; Rushworth et al., 2002), other studies found no effect (Hsieh, 2006; Hsieh \& Yu, 2003; Hsieh \& Liu, 2005; Poulsen et al., 2005). However, all these studies used manual left/right responding in rather simple tasks, such as switching between color categorization (red, blue) and letter categorization (vowel, consonant). Thus, even if we take these earlier studies to suggest that switching modulates the amplitude of the P300, it would remain an open question whether previous results obtained with binary manual tasks (i.e., pressing left or right buttons) generalize to other more complex tasks such as spoken noun-phrase production. In the present study, switching between phrase types did not modulate the P300 amplitude, suggesting that previous results from manual tasks do not straightforwardly generalize to spoken phrase production.
To summarize, we found evidence that producing long phrases prolongs RTs and reduces the P300 compared to producing short phrases. The larger the P300 effect, the smaller the RT effect. This suggests that a greater engagement of the updating ability leads to a smaller RT difference between long and short phrases.

\subsection{Conclusion}

The present study provided evidence on the electrophysiological basis and dynamics of the involvement of updating, inhibiting, and shifting abilities in noun-phrase production. In picture description RTs, length, distractor, and switch effects were obtained, which have been associated with the updating, inhibiting, and shifting abilities. A switch effect was obtained in the N200, widely distributed across the scalp, and length effects were found in the anterior and posterior $\mathrm{N} 200$, and in the P300. Moreover, length and switch interacted in the posterior N200. We argued that these findings suggest that inhibiting and shifting of task set occur before updating in phrase planning.

\section{References}

Allport, A., Wylie, G., 1999. Task-switching: Positive and negative priming of taskset. In: Humphreys, G.W., Duncan, J., Treisman, A. (Eds.), Attention, Space, and Action: Studies in Cognitive Neuroscience. Oxford University Press, Oxford, pp. 273-296.

Allport, A., Wylie, G., 2000. 'Task-switching', stimulus-response bindings, and negative priming. In: Monsell, S., Driver, J.S. (Eds.), Attention and Performance XVIII: Control of Cognitive processes. MIT Press, Cambridge, MA.

Aristei, S., Melinger, A., Abdel Rahman, R., 2011. Electrophysiological chronometry of semantic context effects in language production. Journal of Cognitive Neuroscience 23, 1567-1586.

Baddeley, A.D., 1996. Exploring the central executive. Quarterly Journal of Experimental Psychology 49A, 5-28.

Brydges, C.R., Fox, A.M., Reid, C.L., Anderson, M., 2014. Predictive validity of the N2 and P3 ERP components to executive functioning in children: A latent-variable analysis. Frontiers in Human Neuroscience 8, 80.

Conway, A.R.A., Kane, M.J., Bunting, M.F., Hambrick, D.Z., Wilhelm, O., Engle, R.W., 2005. Working memory span tasks: A methodological review and user's guide. Psychonomic Bulletin \& Review 12, 769-786.

Evans, J.L., Selinger, C., Pollak, S.D., 2011. P300 as a measure of processing capacity in auditory and visual domains in specific language impairment. Brain Research 1389, 93-102.

Falkenstein, M., Hoormann, J., Hohnsbein, J., 1999. ERP components in go/nogo tasks and their relation to inhibition. Acta Psychologica 101, 267-291.

Folstein, J.R., Van Petten, C., 2008. Influence of cognitive control and mismatch on the N2 component of the ERP: A review. Psychophysiology 45, 152-170.

Gilbert, S.J., Burgess, P.W., 2008. Executive function. Current Biology 18, R110-R114.

Gilbert, S.J., Shallice, T., 2002. Task switching: A PDP model. Cognitive Psychology 44, 297-337.

Habets, B., Jansma, B.M., Münte, T.F., 2008. Neurophysiological correlates of linearization in language production. BMC Neuroscience 9, article 77.

Heil, M., Osman, A., Wiegelmann, J., Rolke, B., Hennighausen, E., 2000. N200 in the Eriksen-task: Inhibitory executive process? Journal of Psychophysiology 14, $218-225$.

Holmes, A.J., Pizzagalli, D.A., 2008. Response conflict and frontocingulate dysfunction in unmedicated participants with major depression. Neuropsychologia 46, 2904-2913.

Hsieh, S., 2006. The lateralized readiness potential and P300 of stimulus-set switching. International Journal of Psychophysiology 60, 284-291.

Hsieh, S., Yu, Y.T., 2003. Exploring the nature of switch cost: Inferences from P300 and the lateralized readiness potentials. Brain Research Protocols 12, 49-59.

Hsieh, S., Liu, L.C., 2005. The nature of switch cost: Task set reconfiguration or carry-over effect? Cognitive Brain Research 22, 165-175.

Jackson, G.M., Swainson, R., Cunnington, R., Jackson, S.R., 2001. ERP correlates of executive control during repeated language switching. Bilingualism: Language and Cognition 4, 169-178.

Jodo, E., Kayama, Y., 1992. Relation of a negative ERP component to response inhibition in a go/no-go task. Electroencephalography and Clinical Neurophysiology 82, 477-482.

Karayanidis, F., Coltheart, M., Michie, P.T., Murphy, K., 2003. Electrophysiological correlates of anticipatory and poststimulus components of task switching. Psychophysiology 40, 329-348.

Koch, I., Gade, M., Schuch, S., Philipp, A.M., 2010. The role of task inhibition in task switching: A review. Psychonomic Bulletin and Review 17, 1-14.

Kok, A., 2001. On the utility of P3 amplitude as a measure of processing capacity. Psychophysiology 38, 557-577.

Kopp, B., Rist, F., Mattler, U., 1996. N200 in the flanker task as a neurobehavioral 
tool for investigating executive control. Psychophysiology 33, 282-294.

Kramer, A.F., Sirevaag, E.J., Braune, R., 1987. A psychophysiological assessment of operator workload during simulated flight missions. Human Factors 29 $145-160$.

Levelt, W.J.M., Roelofs, A., Meyer, A.S., 1999. A theory of lexical access in speech production. Behavioral and Brain Sciences 22, 1-38.

Levelt, W.J.M., 1989. Speaking: From Intention to Articulation. MIT Press, Cambridge, MA.

Logan, G.D., 1985. Executive control of thought and action. Acta Psychologica 60, 193-210.

Lorist, M.M., Klein, M., Nieuwenhuis, S., De Jong, R., Mulder, G., Meijman, T.F., 2000 Mental fatigue and task control: Planning and preparation. Psychophysiology 37, 614-625.

Marek, A., Habets, B., Jansma, B.M., Nager, W., Münte, T.F., 2007. Neural correlates of conceptualisation difficulty during the preparation of complex utterances. Aphasiology 21, 1147-1156.

Meuter, R.F.I., Allport, A., 1999. Bilingual language switching in naming: Asymmetrical costs of language selection. Journal of Memory and Language 40, 25-40.

Miyake, A., Friedman, N.P., Emerson, M.J., Witzki, A.H., Howerter, A., Wager, T., 2000. The unity and diversity of executive functions and their contributions to complex "frontal lobe" tasks: A latent variable analysis. Cognitive Psychology $41,49-100$.

Piai, V., Roelofs, A., 2013. Working memory capacity and dual-task interference in picture naming. Acta Psychologica 142, 332-342.

Polich, J., 2007. Updating P300: An integrative theory of P3a and P3b. Clinical Neuropsychology 118, 2128-2148.

Polich, J., Kok, A., 1995. Cognitive and biological determinants of P300: An integrative review. Biological Psychology 41, 103-146.

Poulsen, C., Luu, P., Davey, C., Tucker, D.M., 2005. Dynamics of task sets: Evidence from dense-array event-related potentials. Cognitive Brain Research 24, 133-154.

Posner, M.I., 2012. Attention in a Social World. Oxford University Press, Oxford, UK. Roelofs, A., 2003. Goal-referenced selection of verbal action: Modeling attentional control in the Stroop task. Psychological Review 110, 88-125.

Roelofs, A., Piai, V., 2011. Attention demands of spoken word planning: A review. Frontiers in Psychology, 2, Article, 307.

Rushworth, M.F.S., Passingham, R.E., Nobre, A.C., 2002. Components of switching intentional set. Journal of Cognitive Neuroscience 14, 1139-1150.

Schmajuk, M., Liotti, M., Busse, L., Woldorff, M.G., 2006. Electrophysiological activity underlying inhibitory control processes in normal adults. Neuropsychologia 44, 384-395.

Schmitt, B.M., Münte, T.F., Kutas, M., 2000. Electrophysiological estimates of the time course of semantic and phonological encoding during implicit picture naming. Psychophysiology 37, 473-484.

Schmiedek, F., Hildebrandt, A., Lövdén, M., Wilhelm, O., Lindenberger, U., 2009.
Complex span versus updating tasks of working memory: The gap is not that deep. Journal of Experimental Psychology: Learning, Memory, and Cognition 35, 1089-1096.

Schnur, T.T., Schwartz, M.F., Kimberg, D., Hirshorn, E., Coslett, H.B., ThompsonSchill, S.L., 2009. Localizing interference during naming: Convergent neuroimaging and neuropsychological evidence for the function of Broca's area. Proceedings of the National Academy of Sciences 106, 322-327.

Shao, Z., Roelofs, A., Acheson, D.J., Meyer, A.S., 2014. Electrophysiological evidence that inhibition supports lexical selection in picture naming. Brain Research 1586, 130-142.

Shao, Z., Roelofs, A., Meyer, A.S., 2012. Sources of individual differences in the speed of naming objects and actions: The contribution of executive control. Quarterly Journal of Experimental Psychology 65, 1927-1944.

Sikora, K., Roelofs, A., Hermans, D., Knoors, H., 2015. Executive control in spoken noun-phrase production: Contributions of updating, inhibiting, and shifting. The Quarterly Journal of Experimental Psychology.

Silton, R., Heller, W., Towers, D., Engels, A., Spielberg, J., Edgar, J., Sass, S., Stewart, J., Sutton, B., Banich, M. Miller, G., 2010. The time course of activity in dorsolateral prefrontal cortex and anterior cingulate cortex during top-down attentional control. NeuroImage 50, 1292-1302.

Strayer, D.L., Drews, F.A., 2007. Cell-phone-induced driver distraction. Current Directions in Psychological Science 16, 128-131.

Van Casteren, M., Davis, M.H., 2006. Mix, a program for pseudorandomization. Behavior Research Methods 38, 584-589.

Verbruggen, F., Logan, G.D., Stevens, M.A., 2008. STOP-IT: Windows executable software for the stop-signal paradigm. Behavior Research Methods 40, 479-483.

Verhoef, K., Roelofs, A., Chwilla, D., 2009. Role of inhibition in language switching: Evidence from event-related brain potentials in overt picture naming. Cognition 110, 84-99.

Verhoef, K., Roelofs, A., Chwilla, D., 2010. Electrophysiological evidence for endogenous control in switching attention between languages in overt picture naming. Journal of Cognitive Neuroscience 22, 1832-1843.

Watter, S., Geffen, G.M., Geffen, L.B., 2001. The n-back as a dual task: P300 morphology under divided attention. Psychophysiology 38, 998-1003.

Wickens, C., Kramer, A., Vanasse, L., Donchin, E., 1983. Performance of concurrent tasks: A psychophysiological analysis of the reciprocity of information-processing resources. Science 221, 1080-1082.

Yeung, N., Monsell, S., 2003. Switching between tasks of unequal familiarity: The role of stimulus-attribute and response-set selection. Journal of Experimental Psychology: Human Perception and Performance 29, 455-469.

Zubicaray, de, G.I., Wilson, S.J., McMahon, K.J., Muthiah, K., 2001. The semantic interference effect in the picture-word paradigm: An event-related fMRI study employing overt responses. Human Brain Mapping 14, 218-227. 